\title{
Elongator: An Ancestral Complex Driving Transcription and Migration through Protein Acetylation
}

\author{
Catherine Creppe and Marcus Buschbeck \\ Chromatin and Cell Fate Group, Institute for Predictive and Personalized Medicine of Cancer (IMPPC), 08916 Badalona, Spain
}

Correspondence should be addressed to Marcus Buschbeck, mbuschbeck@imppc.org

Received 14 July 2010; Accepted 5 December 2010

Academic Editor: Patrick Matthias

Copyright $\odot 2011$ C. Creppe and M. Buschbeck. This is an open access article distributed under the Creative Commons Attribution License, which permits unrestricted use, distribution, and reproduction in any medium, provided the original work is properly cited.

\begin{abstract}
Elongator is an evolutionary highly conserved complex. At least two of its cellular functions rely on the intrinsic lysine acetyltransferase activity of the Elongator complex. Its two known substrates-Histone $\mathrm{H} 3$ and $\alpha$-Tubulin-reflect the different roles of Elongator in the cytosol and the nucleus. A picture seems to emerge in which nuclear Elongator could regulate the transcriptional elongation of a subset of stress-inducible genes through acetylation of Histone $\mathrm{H} 3$ in the promoter-distal gene body. In the cytosol, Elongator-mediated acetylation of $\alpha$-Tubulin contributes to intracellular trafficking and cell migration. Defects in both functions of Elongator have been implicated in neurodegenerative disorders.
\end{abstract}

\section{Introduction}

Elongator was initially identified in yeast as part of the RNA polymerase II (RNAPII) holoenzyme [1]. Since this interaction was dependent on the hyperphosphorylation of the C-terminal repeats of RNAPII, which is a mark of transcriptional elongation, the complex was termed Elongator. The fully functional Elongator complex consists of six subunits [2-4] and has a physiologically important role. This is for instance demonstrated by several observations. First, loss of Elongator function in yeast provokes hypersensitivity to stresses including elevated temperature and osmotic conditions [2, 5]. Second, in Arabidopsis, mutations in Elongator result in growth defects [6]. Moreover, mice mutations in Elongator subunits are embryonic lethal [7], and finally, impaired Elongator activity has been correlated with familial dysautonomia in human patients [8].

Both yeast and human Elongator have lysine acetyltransferase (KAT) activity $[5,9,10]$. Only two main substrates for Elongator have been identified: Histone $\mathrm{H} 3$ and $\alpha$-Tubulin. These two different substrates reflect the different functions of Elongator complex in the nucleus and in the cytosol. After having been identified in the context of transcription, it was first surprising to find that the major fraction of the Elongator complex indeed resided in the cytosol [912]. While in the nucleus acetylation of Histone $\mathrm{H} 3$ is linked to the function of Elongator in transcription [13], cytosolic acetylation of $\alpha$-Tubulin by Elongator has been recently connected to microtubules function particularly in the context of cell migration [14].

Other functions of Elongator have also been described. These include roles in tRNA processing $[15,16]$ and exocytosis [17], which have been reviewed elsewhere [13, 18]. Here, we will focus on the Elongator functions for which the involvement of protein acetylation has been well established. In particular, we will discuss similarities and differences in its molecular mode of function in the nucleus and cytosol. We will further discuss the link between the dysfunction of the Elongator complex and neurological disorders.

\section{Biochemical and Other Aspects of Elongator}

Elongator was first copurified with yeast RNAPII as three subunits containing complex [1]. Direct affinity purifications, however, made clear that the functional unit of Elongator contains six subunits that can be separated in two stable sub-complexes under high-salt conditions [2-4]. 
The subunits of these two complexes were termed Elp13 and Elp4-6. Although these proteins have been given many other names depending on the contexts of their initial identification, for simplification, here we will stick to the Elp nomenclature.

All Elp proteins are evolutionary highly conserved which is best illustrated by cross-species rescue experiments. Arabidopsis Elp1 could rescue deficient yeast [19], and human Elp1 could rescue Elp1-deficient neurons in mouse cortex [7]. Human Elp3 and Elp4 could further compensate for the lack of their counterparts in yeast [20].

Within the Elongator complex, the $150 \mathrm{kDa}$ protein Elp1 is the largest subunit. It contains several WD40 repeats and is considered to mainly function as scaffold protein, which is required for the formation of the complex [21]. More recently, however, Elp1 proteins from yeast, C. elegans, and human were shown to possess RNA-dependent RNA polymerase activity [22]. Elp2 also contains WD40 domain [23]. In contrast to Elp1, however, Elp2 does not seem to provide any scaffolding function since the remaining complex could form in its absence [24]. The Elp3 subunit of the complex possesses motifs characteristic of the GCN5 histone acetyl transferase family [5] and was shown to be able to acetylate histones [25]. In addition, Elp3 contains an iron-sulfur cluster, which can bind S-adenosylmethionine [26] and which is essential for the structural integrity of the Elongator complex [27]. It was first suggested that this domain could have catalytic activity and function as histone demethylase [28], but experimental studies failed to confirm this hypothesis. Instead, recent evidence suggests that Elongator might be involved in DNA demethylation [30]. The integrity of Elongator, and more particularly the iron-sulfur cluster of Elp3, was shown to be essential for the global zygotic DNA demethylation occurring on the paternal genome at the postfertilization stage [30]. On the basis of sequence comparisons, it was suggested that Elp4 and Elp6 could be inactive orthologues of ancestral ATPases involved in chromatin remodeling [31].

Combining genetic inactivation and affinity purifications $[11,21]$, Frohloff and colleagues were the first to put forward a model for the architecture of the hexameric Elongator complex that was largely confirmed by others [32]. While efforts to determine the three-dimensional structure of the Elongator complex are still under way, the model can serve as a good basis for our experimental design. As shown in Figure 1, Elp1 is the central subunit that mediates the binding of the two subcomplexes. On one side, Elp1 directly interacts with Elp3, which in turn binds Elp2. On the other hand, Elp1 also directly binds the Elp4-6 subcomplex through Elp4. Whether Elp6 and Elp5 also directly contact Elp1 is less clear at present.

The functional unit of Elongator is the holocomplex containing all six subunits. This is best illustrated in yeast where strains lacking any of the 6 Elp proteins have very similar phenotypes. For instance, they display a particular "slow start" phenotype as manifested by a pronounced delay in adaptation to new growth medium $[1,4]$. Elp-deficient strains are further temperature, salt, and caffeine sensitive $[2,4]$ and resistant to the K. lactis killer toxin [3, 33]. There
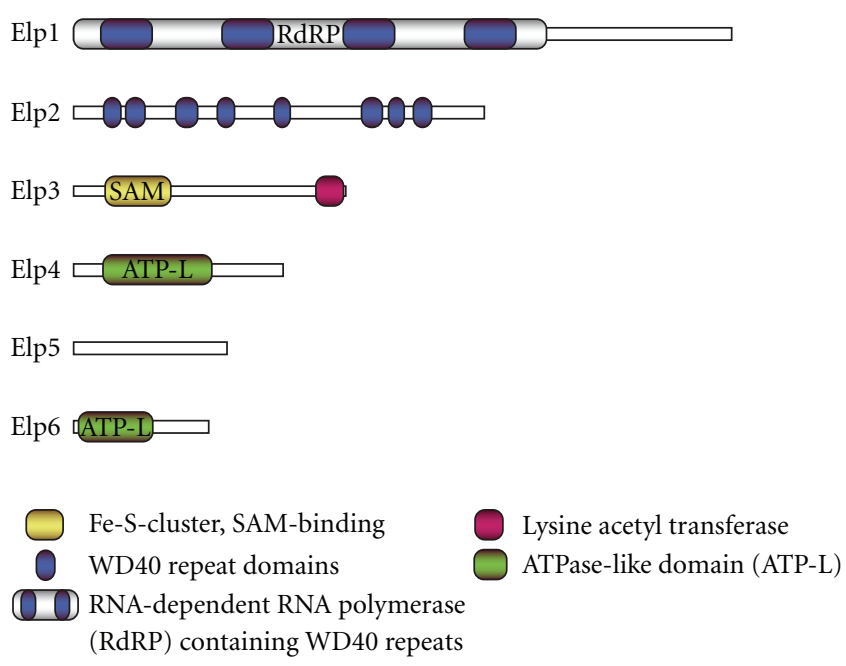

(a)

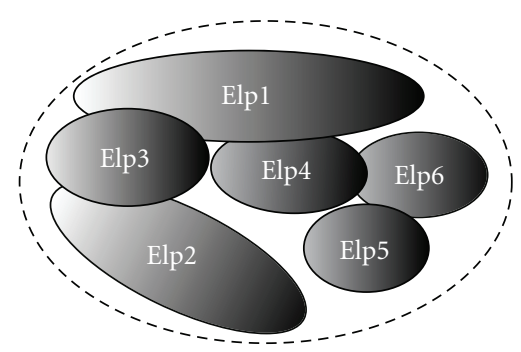

(b)

Figure 1: The Elongator complex and its components. (a) Schematic view of the Elp proteins and their domain structure. (b) Current model for the architecture of the Elongator complex.

is still some ambiguity in the questions whether elp-mutant strains are sensitive to the pyrimidine analogue 6-azauracil [1-3]. Most importantly, KAT activity of Elongator was essential for the rescue of all phenotypes in yeast. Removal of almost any of the Elongator subunits affects the interaction of the others $[11,21,24,32]$ and might explain why all subunits are functionally essential in vivo. In line with this, it could be demonstrated that Elongator requires all its subunits for efficient KAT activity [34].

Several proteins have been reported to interact with components of the Elongator complex. These include Kti12 [33], IKB [35, 36], STAT3 [36], and PCNA [32]. Although some interactions have been confirmed in different species, most have only been found in a single species. It remains to be seen which interactions are as conserved as Elongator itself and which have evolved later. Mainly from studies focusing on zymocin resistance in yeast, for instance, comes the statement that Elongator interacts with the Hrr kinase and the Sit 4 phosphatase $[37,38]$. In a series of publications, the authors establish that these interactions result in cycles of phosphorylation and dephosphorylation events that are essential for Elongator function in zymocin resistance. Whether such a phosphorylation-dependent control is also 
relevant for the other functions of Elongator remains to be elucidated. In addition, several interactions that have been found by one group could so far not be confirmed by others, which suggests that many of these interactions could be transient and might thus only occur under distinct conditions. These "potential" interactors are not further discussed here.

\section{Acetylation of Histones: Elongator in Transcription}

A surprising large number of genes, which can be induced by external stimuli, are regulated at the level of transcriptional elongation $[39,40]$. These genes include stress-inducible genes as well as genes activated during developmental processes. For these genes, RNAPII is stalled or paused in close proximity to the promoters in the absence of the stimuli. Upon stimulation, the C-terminal domain of RNAPII becomes hyperphosphorylated and resumes transcription whose procession is controlled by elongation factors. These elongation factors can be divided into active and passive factors in respect to whether or not they directly affect enzymatic activity of RNAPII [41]. After several years of controversy that followed the initial copurification of the Elongator with transcription-competent RNAPII [1], today there is little doubt that one of the functions of Elongator is indeed transcriptional elongation. However, the exact mode of action is still not fully understood. Also the debate whether the Elongator should be considered as active or passive elongation factor is still open. Here, we review the data of the last ten years that link Elongator to transcriptional elongation.

The observation that Elp3 subunit is similar to the GCN5 suggested early that the Elongator complex could be a histone acetyltransferase [5]. In vitro, purified Elp3 was able to acetylate both $\mathrm{H} 3$ and $\mathrm{H} 4$ [5], and in vivo, a mutant lacking its putative KAT activity had a similar phenotype as complete loss of Elp3 [25, 33]. Synthetic lethality experiments further provided the first evidence pointing towards Histone $\mathrm{H} 3$ and in particular Lysine 14 as main in vivo target of Elp3 [25]. The functionality of Elongator's KAT activity and its specificity for Histone $\mathrm{H} 3$ were later confirmed in several species including yeast, plants, and mammalian cells [34, $42,43]$. In vitro, Elongator was able to enhance RNAPII transcription from a chromatinic template in an Acetyl-CoAdependent manner [10], while, on naked DNA, Elongator had no influence on RNAPII transcription $[2,10]$.

The target genes of Elongator depend a lot on the biologic system studied. In yeast, loss of Elongator function reduced the activation of a number of inducible genes $[1,2,5]$ but did not affect transcription globally [2]. In plants, Elongator contributes to the transcription of stress-induced and auxinrelated genes [19, 43]. In human cells, Elongator was required for the activation of several genes involved in migration [42] and for the activation of HSP70 in heat shock conditions [29]. Elongator was also involved in regulation of several p53-dependent proapoptotic genes but in a manner that did not seem to occur on the transcriptional level [44].
In line with a possible function for Elongator in regulating these genes by facilitating their transcriptional elongation, several groups could now demonstrate that Elongator preferentially occupies the open reading frames but not the promoters of these genes [29, 42]. Indeed, Elongator binding was mostly observed several kilobases downstream of the transcriptional start site (TSS) [45]. In yeast, Elongator could be cross-linked to the nascent pre-mRNAs of regulated genes [46]. In line with the initial purification of Elongator with the hyperphosphorylated form of RNAPII [1], timecourse experiments suggested that Elongator indeed arrives at genes after hyperphosphorylation of RNAPII [47]. It has to be pointed out that Elongator could also be found on genes whose transcription was not affected by its depletion suggesting some functional redundancy of Elongator with other elongation factors. Taking the function of Elongator in the context of chromatin into account, it is the most similar to the FACT complex. FACT was suggested to regulate Elongation by facilitating the procession of RNAPII through nucleosomes [41]. In human cells, both FACT and Elongator could be found on a common gene, where in contrast to Elongator, FACT was bound at the more TSS-proximal regions of the ORF [45]. This observation provokes the intriguing speculation that FACT and Elongator could have distinct functions in the early and late elongation process. It is further interesting to note that genetic inactivation of both FACT and Elongator resulted in synthetic lethality in yeast [48]. Whether Elongator facilitates the opening and reconstitution of nucleosomes in a FACT-like manner remains to be investigated.

Taken together, a picture emerges in which Elongator regulates the transcriptional elongation of a subset of stress-inducible genes through acetylation of Histone $\mathrm{H} 3$ in the TSS distal gene body (see Figure 2). Although the elongation function of Elongator is conserved, the subset of regulated genes has diverged during evolution. Many open questions remain such as how is the target gene specificity of Elongator determined? What is the molecular consequence of Elongator-mediated histone acetylation? Considering the other functions of Elongator, manipulation of its function is likely to affect indirect as well as direct target genes. Hence, it will inevitable to check for the presence of Elongator at putative target genes when addressing the role of Elongator in transcription.

\section{Acetylation of Tubulin: Elongator and Cellular Motility}

The cytoskeleton is characterized by its dynamic structure and is composed of three types of fibers: actin filaments, intermediate filaments, and microtubules [49]. The functions of the cytoskeleton go far beyond the maintenance of cell shape and include important roles in cellular motility, intracellular transport, and mitosis [50].

Microtubules are the biggest cytoskeletal fibers that are essentially composed of only two proteins, the $\alpha$ - and $\beta$ Tubulins [51]. Heterodimers of globular $\alpha$ - and $\beta$-Tubulins polymerize in a head-to-tail fashion to form the so-called 


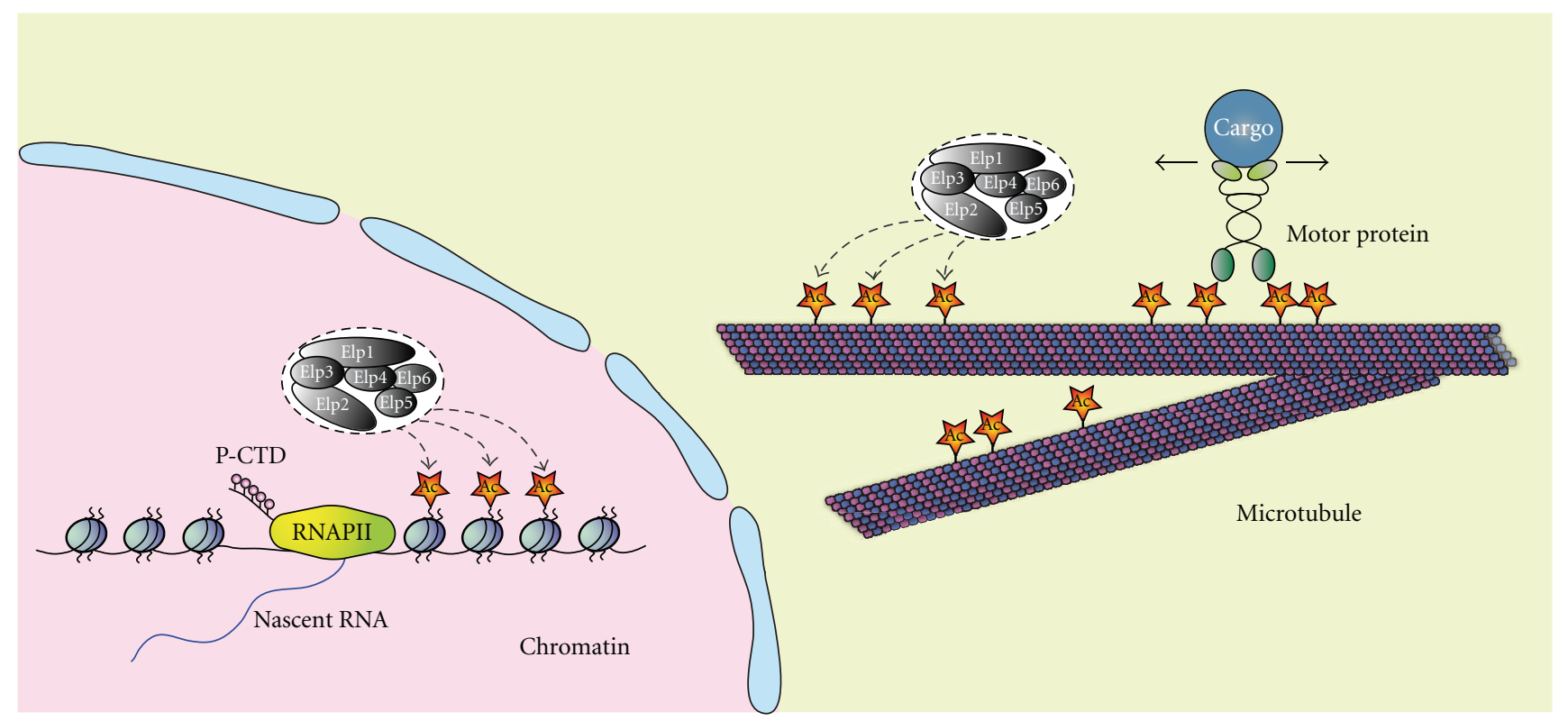

FIGURE 2: Two acetylation-dependent functions of Elongator. In the nucleus, Elongator-mediated acetylation of Histone H3 contributes to transcriptional elongation. Thereby, it interacts with processive RNAPII, which is hyperphosphorylated at its C-terminal domain (CTD). Although this function of Elongator is conserved from yeast to man, the subset of genes that underly this kind of regulation have diverged during evolution. In the cytosol, Elongator acetylates the $\alpha$-Tubulin subunit of microtubules. This acetylation event contributes to the trafficking of cargo along microtubules and cell migration. Please note that acetylation of $\alpha$-Tubulin occurs in the lumen of microtubule, but for reasons of illustration, it has been depicted on outside here.

protofilaments. Of these protofilaments, 13 bind to each other in a parallel way to form the tubular structure of the microtubule, which has a diameter of about $25 \mathrm{~nm}$. An important characteristic of these microtubules is their polarity. The early in vitro observation that new heterodimers added faster to one end of the microtubules leads to the distinction of plus and minus ends [52, 53]. Minus ends terminate in $\alpha$-Tubulin subunits and plus-ends accordingly in $\beta$-Tubulin subunits. By establishing and maintaining cell polarity, the remodelling of microtubules is particularly important in directed cell migration processes $[54,55]$.

Several PTMs occur on Tubulins that are considered to modulate the function of microtubules by providing binding sites for regulatory proteins [56]. These PTMs include tyrosination, polyglycylation, and polyglutamylation. Considering the tubular architecture of microtubuli it is not surprising that most PTMs occur on the carboxyterminal domains of both $\alpha$ - and $\beta$-Tubulins, which are exposed on the outer surface of the tube [57].

Additionally, $\alpha$-Tubulin but not $\beta$-Tubulin is acetylated in vivo [58]. The particularity of this PTM is its localization. The acetylation of $\alpha$-Tubulin, which exclusively occurs on lysine 40 , is located on the luminal inner surface of the microtubule [59]. While HDAC6 and Sirt2 have been described a few years ago as tubulin deacetylases [60-62], the responsible acetylase remained elusive. Just recently, the Elp3 subunit of cytosolic Elongator was identified as $\alpha$ Tubulin-specific KAT in human and mouse cells [14]. It could be shown by mutating a critical residue in the catalytic domain of Elp3 that Elongator is responsible for a substantial amount of tubulin acetylation [14]. Importantly, acetylation of tubulin by Elongator does also occur in the nematode $C$. elegans [63] suggesting a similar high degree of conservation for the cytosolic function of Elongator as previously shown for its nuclear role in transcription. The identification of MEC-17 as a second $\alpha$-Tubulin acetyltransferase made clear that Elongator is not the only one [64]. Indeed, in Tetrahymena and neurons from zebrafish embryos and C. elegans hermaphrodites, MEC-17 was shown to be required for most if not all $\alpha$-Tubulin acetylation. We can expect additional acetyltransferases to be identified in the future, and the task before the scientific community will be to sort out the context-dependent contributions of each of these enzymes.

Acetylation of microtubules seems not to be essential for cell survival but plays a role in cell motility and in motor-based trafficking $[65,66]$. The role of acetylation in cell motility was first demonstrated by several studies reporting that HDAC6 overexpression that promotes $\alpha$ Tubulin deacetylation increases cell motility whereas HDAC6 inhibition triggers the opposite effect $[60,67]$. The migration defects observed in neurons depleted for Elongator subunits confirmed the important function of tubulin acetylation during motility processes $[14,63]$. Similarly, the loss of the other acetyltransferase MEC-17 reduced the touch sensitivity of neurons in C. elegans and induced phenotypes consistent with neuromuscular defects in zebrafish embryos [64].

However, what is the molecular mechanism by which acetylation of tubulin affects cellular motility? Some clues came from studies of motor proteins that transport diverse cargoes along microtubules. Microtubule-specific motor 
proteins are divided into two classes called dyneins and kinesins depending on the direction of their movement $[68,69]$. Most of the members of the kinesin superfamily move to the plus end of microtubules, whereas members of the dynein superfamily transport their cargoes to the minus end $[70,71]$.

Acetylation of $\alpha$-Tubulin was shown to promote in particular the binding and the motility of kinesin-1 [66]. In the cerebral cortex, Elongator mediated the acetylation of microtubules and could thereby direct anterograde transport of defined cargoes to growing neurites in developing neurons and to axons in mature cells [18]. Acetylation of $\alpha$ Tubulin was also associated recently to the posttranslational fine tuning of $\alpha$-Tubulin levels and to the dynamics of polymerization and depolymerization [63]. Both transport and changes in the microtubule dynamics could directly influence the cellular motility.

With the identification of $\alpha$-Tubulin as cytosolic target for the Elongator's KAT activity, a new chapter was opened. Many questions persist. For instance, it is not clear how Elongator or the counteracting deacetylases HDAC6 and SirtT2 can access lysine 40 at its luminal position. It is further still enigmatic how a luminal modification can influence the motor protein functions that occur on the cytoplasmic surface of the microtubule. How are the activities of aTubulin-specific acetylases regulated? It is interesting to point out that the level of $\alpha$-Tubulin acetylation is quite variable. In neurons, for instance, the large majority of cellular $\alpha$-Tubulin is acetylated.

\section{Defects in Elongator and Neurodegenerative Disorders}

Elongator is associated to different neuronal diseases. While the familial dysautonomia is associated to mutation of the IKBKAP gene encoding Elp1 $[8,72]$, the amyotrophic lateral sclerosis was recently shown to be linked to allelic variants of ELP3 [73].

Familial dysautonomia (FD), also known as Riley-Day syndrome, is characterized by a progressive depletion of unmyelinated sensory and autonomic neurons resulting in a loss of neuronal function which leads to a complex symptomatology including gastrointestinal dysfunction, lack of overflow tears, absence of fungiform papillae on the tongue and other autonomic disturbances [74]. The major genetic cause of FD is a single noncoding mutation of the Elp1 encoding gene which affects its splicing. Interestingly, this mutation is not fully penetrant, which means that some residual amounts of the wild-type Elp1 (Elp1-WT) mRNA are nevertheless synthesized. The central nervous and the peripheral nervous systems are the major tissues affected by this splicing defect as demonstrated by the low level of WT-Elp1 present in these tissues [75]. Amyotrophic lateral sclerosis (ALS) is caused by the degeneration of motor neurons resulting in muscle weakness and atrophy. Affected patients show cognitive impairments and usually die from respiratory muscle weakness. Defects in Elongator have already been implicated in the pathogenesis of ALS [73].
The observation that alterations of Elongator correlate with two different neurodegenerative disorders suggests that Elongator has an essential function in the development and the maintenance of neuronal networks. On the molecular level, Elongator exerts its "neuronal" function at least partially through acetylation of its two main substratesHistone $\mathrm{H} 3$ and $\alpha$-Tubulin. On one hand, defective Elongator could reduce the elongation of gene transcripts that normally mediate the motility, the development, and the survival of neuronal cells [42]. On the other hand, defects in intracellular trafficking have already been linked to several neurodegenerative diseases including Alzheimer's disease, Parkinson, disease, or ALS [76-78]. Thus, it is reasonable to speculate that the loss of Elongator-mediated tubulinacetyation could result in defective intracellular transport of cargo and thereby contribute to neurodegeneration, but we have also to point out that Elongator might have other yet unknown substrates that could contribute to the symptomatology of FD and ALS.

\section{Final Considerations and Outlook}

The two substrates of Elongator's acetylase activity- $\alpha$ Tubulin and Histone $\mathrm{H} 3$ - are as different as the functions of Elongator in the cytosol and the nucleus, or, do they have anything in common? Well, both $\alpha$-Tubulin and Histone H3 are main components of cellular macrocomplexes: microtubules and chromatin. Both microtubules and chromatin are formed by repetitive units. We can speculate that Elongator might act as a processive enzyme in which each acetylation reaction would facilitate the next. This would predict that Elongator has a preference for carrying out sequential reactions. Experimentally, this could be tested in acetylation assays by comparing single tubulin dimers and microtubules. For both microtubules and chromatin it is evident that Elongator is not the only acetylating enzyme. In both cases acetylation has further been suggested to be one of many modifications that together could form a type of code. According to the histone or tubulin code hypotheses $[79,80]$, the combination of modifications would be read and interpret by factors that ultimately regulate the function of chromatin and microtubules, respectively.

It was known that Elongator has also other acetylationindependent functions in secretion and tRNA processing [13]. Recent evidences further linked Elongator to the DNAdamage response and gene silencing [32], RNA interference [22], and global DNA demethylation [30]. It looks like a large number of cellular functions have evolved in parallel that make use of the same highly conserved Elongator complex albeit in very different contexts. It remains to be seen which functions of Elongator require its acetylase activity and what would be the corresponding substrates. We are still far from having a comprehensive view of all Elongator functions.

\section{Acknowledgments}

The authors thank Jesper Svejstrup, Laurent Nguyen, and members from the Buschbeck lab for critically reading the 
manuscript. Research in the Buschbeck lab is supported by a SAF2009 and a RYC2010 grant from the Spanish Ministry of Science and Innovation. Catherine Creppe is supported by a postdoctoral FEBS long-term fellowship and Marcus Buschbeck holds a Ramón y Cajal position.

\section{References}

[1] G. Otero, J. Fellows, LI. Yang et al., "Elongator, a multisubunit component of a novel RNA polymerase II holoenzyme for transcriptional elongation," Molecular Cell, vol. 3, no. 1, pp. 109-118, 1999.

[2] N. J. Krogan and J. F. Greenblatt, "Characterization of a sixsubunit Holo-Elongator complex required for the regulated expression of a group of genes in Saccharomyces cerevisiae," Molecular and Cellular Biology, vol. 21, no. 23, pp. 8203-8212, 2001.

[3] Y. Li, Y. Takagi, Y. Jiang et al., "A Multiprotein Complex That Interacts with RNA Polymerase II Elongator," Journal of Biological Chemistry, vol. 276, no. 32, pp. 29628-29631, 2001.

[4] G. Sebastiaan Winkler, T. G. Petrakis, S. Ethelberg et al., "RNA polymerase II elongator holoenzyme is composed of two discrete subcomplexes," Journal of Biological Chemistry, vol. 276, no. 35, pp. 32743-32749, 2001.

[5] B. Ø. Wittschieben, G. Otero, T. De Bizemont et al., "A novel histone acetyltransferase is an integral subunit of elongating RNA polymerase II holoenzyme," Molecular Cell, vol. 4, no. 1, pp. 123-128, 1999.

[6] H. Nelissen, D. Fleury, L. Bruno et al., "The elongata mutants identify a functional Elongator complex in plants with a role in cell proliferation during organ growth," Proceedings of the National Academy of Sciences of the United States of America, vol. 102, no. 21, pp. 7754-7759, 2005.

[7] Y. T. Chen, M. M. Hims, R. S. Shetty et al., "Loss of mouse ikbkap, a subunit of elongator, leads to transcriptional deficits and embryonic lethality that can be rescued by human IKBKAP," Molecular and Cellular Biology, vol. 29, no. 3, pp. 736-744, 2009.

[8] S. A. Slaugenhaupt, A. Blumenfeld, S. P. Gill et al., "Tissuespecific expression of a splicing mutation in the IKBKAP gene causes familial dysautonomia," American Journal of Human Genetics, vol. 68, no. 3, pp. 598-605, 2001.

[9] N. A. Hawkes, G. Otero, G. Sebastiaan Winkler et al., "Purification and characterization of the human elongator complex," Journal of Biological Chemistry, vol. 277, no. 4, pp. 3047-3052, 2002.

[10] J. H. Kim, W. S. Lane, and D. Reinberg, "Human elongator facilitates RNA polymerase II transcription through chromatin," Proceedings of the National Academy of Sciences of the United States of America, vol. 99, no. 3, pp. 1241-1246, 2002.

[11] L. Fichtner, F. Frohloff, D. Jablonowski, M. J. R. Stark, and R. Schaffrath, "Protein interactions within Saccharomyces cerevisiae elongator, a complex essential for Kluyveromyces lactis zymocicity," Molecular Microbiology, vol. 45, no. 3, pp. 817-826, 2002.

[12] D. K. Pokholok, N. M. Hannett, and R. A. Young, "Exchange of RNA polymerase II initiation and elongation factors during gene expression in vivo," Molecular Cell, vol. 9, no. 4, pp. 799809, 2002.

[13] J. Q. Svejstrup, “Elongator complex: how many roles does it play?" Current Opinion in Cell Biology, vol. 19, no. 3, pp. 331336, 2007.
[14] C. Creppe, L. Malinouskaya, M. L. Volvert et al., "Elongator controls the migration and differentiation of cortical neurons through acetylation of $\alpha$-tubulin," Cell, vol. 136, no. 3, pp. 551-564, 2009.

[15] A. Esberg, BO. Huang, M. J. O. Johansson, and A. S. Byström, "Elevated levels of two tRNA species bypass the requirement for elongator complex in transcription and exocytosis," Molecular Cell, vol. 24, no. 1, pp. 139-148, 2006.

[16] BO. Huang, M. J. O. Johansson, and A. S. Byström, "An early step in wobble uridine tRNA modification requires the Elongator complex," RNA, vol. 11, no. 4, pp. 424-436, 2005.

[17] P. B. Rahl, C. Z. Chen, and R. N. Collins, "Elplp, the yeast homolog of the FD disease syndrome protein, negatively regulates exocytosis independently of transcriptional elongation," Molecular Cell, vol. 17, no. 6, pp. 841-853, 2005.

[18] L. Nguyen, S. Humbert, F. Saudou, and A. Chariot, "Elongator-an emerging role in neurological disorders," Trends in Molecular Medicine, vol. 16, no. 1, pp. 1-6, 2010.

[19] Z. Chen, H. Zhang, D. Jablonowski et al., "Mutations in ABO1/ELO2, a subunit of holo-elongator, increase abscisic acid sensitivity and drought tolerance in Arabidopsis thaliana," Molecular and Cellular Biology, vol. 26, no. 18, pp. 6902-6912, 2006.

[20] F. Li, J. Lu, Q. Han, G. Zhang, and B. Huang, "The Elp3 subunit of human Elongator complex is functionally similar to its counterpart in yeast," Molecular Genetics and Genomics, vol. 273, no. 3, pp. 264-272, 2005.

[21] F. Frohloff, D. Jablonowski, L. Fichtner, and R. Schaffrath, "Subunit communications crucial for the functional integrity of the yeast RNA polymerase II elongator ( $\gamma$-toxin target (TOT) ) complex," Journal of Biological Chemistry, vol. 278, no. 2, pp. 956-961, 2003.

[22] C. Lipardi and B. M. Paterson, "Identification of an RNAdependent RNA polymerase in Drosophila involved in RNAi and transposon suppression," Proceedings of the National Academy of Sciences of the United States of America, vol. 106, no. 37, pp. 15645-15650, 2009.

[23] J. Fellows, H. Erdjument-Bromage, P. Tempst, and J. Q. Svejstrup, "The Elp2 subunit of elongator and elongating RNA polymerase II holoenzyme is a WD40 repeat protein," Journal of Biological Chemistry, vol. 275, no. 17, pp. 12896-12899, 2000.

[24] T. G. Petrakis, B. Ø. Wittschieben, and J. Q. Svejstrup, "Molecular architecture, structure-function relationship, and importance of the Elp3 subunit for the RNA binding of holoElongator," Journal of Biological Chemistry, vol. 279, no. 31, pp. 32087-32092, 2004.

[25] B. Ø. Wittschieben, J. Fellows, DU. Wendy, D. J. Stillman, and J. Q. Svejstrup, "Overlapping roles for the histone acetyltransferase activities of SAGA and Elongator in vivo," EMBO Journal, vol. 19, no. 12, pp. 3060-3068, 2000.

[26] C. Paraskevopoulou, S. A. Fairhurst, D. J. Lowe, P. Brick, and S. Onesti, "The Elongator subunit Elp3 contains a FeS cluster and binds S-adenosylmethionine," Molecular Microbiology, vol. 59, no. 3, pp. 795-806, 2006.

[27] C. Greenwood, L. A. Selth, A. Barbara Dirac-Svejstrup, and J. Q. Svejstrup, "An iron-sulfur cluster domain in Elp3 important for the structural integrity of elongator," Journal of Biological Chemistry, vol. 284, no. 1, pp. 141-149, 2009.

[28] Y. Chinenov, "A second catalytic domain in the Elp3 histone acetyltransferases: a candidate for histone demethylase activity?" Trends in Biochemical Sciences, vol. 27, no. 3, pp. 115-117, 2002. 
[29] Q. Han, X. Hou, D. Su et al., "HELP3 subunit of the Elongator complex regulates the transcription of HSP70 gene in human cells," Acta Biochimica et Biophysica Sinica, vol. 39, no. 6, pp. 453-461, 2007.

[30] Y. Okada, K. Yamagata, K. Hong, T. Wakayama, and YI. Zhang, "A role for the elongator complex in zygotic paternal genome demethylation," Nature, vol. 463, no. 7280, pp. 554-558, 2010.

[31] C. P. Ponting, "Novel domains and orthologues of eukaryotic transcription elongation factors," Nucleic Acids Research, vol. 30, no. 17, pp. 3643-3652, 2002.

[32] Q. Li, A. M. Fazly, H. Zhou, S. Huang, Z. Zhang, and B. Stillman, "The elongator complex interacts with PCNA and modulates transcriptional silencing and sensitivity to DNA damage agents," PLoS Genetics, vol. 5, no. 10, Article ID e1000684, 2009.

[33] F. Frohloff, L. Fichtner, D. Jablonowski, K. D. Breunig, and R. Schaffrath, "Saccharomyces cerevisiae Elongator mutations confer resistance to the Kluyveromyces lactis zymocin," EMBO Journal, vol. 20, no. 8, pp. 1993-2003, 2001.

[34] G. S. Winkler, A. Kristjuhan, H. Erdjument-Bromage, P. Tempst, and J. Q. Svejstrup, "Elongator is a histone H3 and $\mathrm{H} 4$ acetyltransferase important for normal histone acetylation levels in vivo," Proceedings of the National Academy of Sciences of the United States of America, vol. 99, no. 6, pp. 3517-3522, 2002.

[35] L. Cohen, W. J. Henzel, and P. A. Baeuerle, "IKAP is a scaffold protein of the I $\kappa$ B kinase complex," Nature, vol. 395, no. 6699, pp. 292-296, 1998.

[36] R. G. Collum, S. Brutsaert, G. Lee, and C. Schindler, "A Stat3-interacting protein (StIP1) regulates cytokine signal transduction," Proceedings of the National Academy of Sciences of the United States of America, vol. 97, no. 18, pp. 10120 10125, 2000.

[37] D. Jablonowski, L. Fichtner, M. J. R. Stark, and R. Schaffrath, "The yeast elongator histone acetylase requires Sit4-dependent dephosphorylation for toxin-target capacity," Molecular Biology of the Cell, vol. 15, no. 3, pp. 1459-1469, 2004.

[38] C. Mehlgarten, D. Jablonowski, K. D. Breunig, M. J. R. Stark, and R. Schaffrath, "Elongator function depends on antagonistic regulation by casein kinase Hrr25 and protein phosphatase Sit4," Molecular Microbiology, vol. 73, no. 5, pp. 869-881, 2009.

[39] G. W. Muse, D. A. Gilchrist, S. Nechaev et al., "RNA polymerase is poised for activation across the genome," Nature Genetics, vol. 39, no. 12, pp. 1507-1511, 2007.

[40] J. Zeitlinger, A. Stark, M. Kellis et al., "RNA polymerase stalling at developmental control genes in the Drosophila melanogaster embryo," Nature Genetics, vol. 39, no. 12, pp. 1512-1516, 2007.

[41] R. J. Sims, R. Belotserkovskaya, and D. Reinberg, "Elongation by RNA polymerase II: the short and long of it," Genes and Development, vol. 18, no. 20, pp. 2437-2468, 2004.

[42] P. Close, N. Hawkes, I. Cornez et al., "Transcription impairment and cell migration defects in elongator-depleted cells: implication for familial dysautonomia," Molecular Cell, vol. 22, no. 4, pp. 521-531, 2006.

[43] H. Nelissen, S. De Groeve, D. Fleury et al., "Plant Elongator regulates auxin-related genes during RNA polymerase II transcription elongation," Proceedings of the National Academy of Sciences of the United States of America, vol. 107, no. 4, pp. 1678-1683, 2010.

[44] I. Cornez, C. Creppe, M. Gillard et al., "Deregulated expression of pro-survival and pro-apoptotic p53-dependent genes upon Elongator deficiency in colon cancer cells," Biochemical Pharmacology, vol. 75, no. 11, pp. 2122-2134, 2008.

[45] A. Kouskouti and I. Talianidis, "Histone modifications defining active genes persist after transcriptional and mitotic in activation," EMBO Journal, vol. 24, no. 2, pp. 347-357, 2005.

[46] C. Gilbert, A. Kristjuhan, G. S. Winkler, and J. Q. Svejstrup, "Elongator interactions with nascent mRNA revealed by RNA immunoprecipitation," Molecular Cell, vol. 14, no. 4, pp. 457464, 2004.

[47] R. Métivier, G. Penot, M. R. Hübner et al., "Estrogen receptor$\alpha$ directs ordered, cyclical, and combinatorial recruitment of cofactors on a natural target promoter," Cell, vol. 115 , no. 6, pp. 751-763, 2003.

[48] T. Formosa, S. Ruone, M. D. Adams et al., "Defects in SPT16 or POB3 (yFACT) in Saccharomyces cerevisiae cause dependence on the Hir/Hpc pathway: polymerase passage may degrade chromatin structure," Genetics, vol. 162, no. 4, pp. 1557-1571, 2002.

[49] S. Etienne-Manneville, "Actin and microtubules in cell motility: which one is in control?" Traffic, vol. 5, no. 7, pp. 470-477, 2004.

[50] G. Liao, T. Nagasaki, and G. G. Gundersen, "Low concentrations of nocodazole interfere with fibroblast locomotion without significantly affecting microtubule level: implications for the role of dynamic microtubules in cell locomotion," Journal of Cell Science, vol. 108, no. 11, pp. 3473-3483, 1995.

[51] K. H. Downing and E. Nogales, "Tubulin structure: insights into microtubule properties and functions," Current Opinion in Structural Biology, vol. 8, no. 6, pp. 785-791, 1998.

[52] T. Horio and H. Hotani, "Visualization of the dynamic instability of individual microtubules by dark-field microscopy," Nature, vol. 321, no. 6070, pp. 605-607, 1986.

[53] T. Mitchinson and M. Kirschner, "Dynamic instability of microtubule growth," Nature, vol. 312, no. 5991, pp. 237-242, 1984.

[54] J. V. Small and I. Kaverina, "Microtubules meet substrate adhesions to arrange cell polarity," Current Opinion in Cell Biology, vol. 15, no. 1, pp. 40-47, 2003.

[55] M. Vicente-Manzanares, D. J. Webb, and A. R. Horwitz, "Cell migration at a glance," Journal of Cell Science, vol. 118, no. 21, pp. 4917-4919, 2005.

[56] K. J. Verhey and J. Gaertig, "The tubulin code," Cell Cycle, vol. 6, no. 17, pp. 2152-2160, 2007.

[57] K. H. Downing and E. Nogales, "Tubulin and microtubule structure," Current Opinion in Cell Biology, vol. 10, no. 1, pp. 16-22, 1998.

[58] S. W. L'Hernault and J. L. Rosenbaum, "Chlamydomonas $\alpha$ tubulin is posttranslationally modified by acetylation on the $\varepsilon$-amino group of a lysine," Biochemistry, vol. 24, no. 2, pp. 473-478, 1985.

[59] M. LeDizet and G. Piperno, "Identification of an acetylation site of Chlamydomonas alpha-tubulin," Proceedings of the National Academy of Sciences of the United States of America, vol. 84, no. 16, pp. 5720-5724, 1987.

[60] C. Hubbert, A. Guardiola, R. Shao et al., "HDAC6 is a microtubule-associated deacetylase," Nature, vol. 417, no. 6887, pp. 455-458, 2002.

[61] Y. Zhang, N. Li, C. Caron et al., "HDAC-6 interacts with and deacetylates tubulin and microtubules in vivo," The EMBO Journal, vol. 22, no. 5, pp. 1168-1179, 2003.

[62] B. J. North, B. L. Marshall, M. T. Borra, J. M. Denu, and E. Verdin, "The human Sir2 ortholog, SIRT2, is an NAD+dependent tubulin deacetylase," Molecular Cell, vol. 11, no. 2, pp. 437-444, 2003. 
[63] J. A. Solinger, R. Paolinelli, H. Klöß et al., "The Caenorhabditis elegans elongator complex regulates neuronal $\alpha$-tubulin acetylation,” PLoS Genetics, vol. 6, no. 1, Article ID e1000820, 2010.

[64] J. S. Akella, D. Wloga, J. Kim et al., "MEC-17 is an $\alpha$-tubulin acetyltransferase," Nature, vol. 467, no. 7312, pp. 218-222, 2010.

[65] J. P. Dompierre, J. D. Godin, B. C. Charrin et al., "Histone deacetylase 6 inhibition compensates for the transport deficit in Huntington's disease by increasing tubulin acetylation," Journal of Neuroscience, vol. 27, no. 13, pp. 3571-3583, 2007.

[66] N. A. Reed, D. Cai, T. L. Blasius et al., "Microtubule acetylation promotes kinesin-1 binding and transport," Current Biology, vol. 16, no. 21, pp. 2166-2172, 2006.

[67] S. J. Haggarty, K. M. Koeller, J. C. Wong, C. M. Grozinger, and S. L. Schreiber, "Domain-selective small-molecule inhibitor of histone deacetylase 6 (HDAC6)-mediated tubulin deacetylation," Proceedings of the National Academy of Sciences of the United States of America, vol. 100, no. 8, pp. 4389-4394, 2003.

[68] S. A. Endow, "Microtubule motors in spindle and chromosome motility," European Journal of Biochemistry, vol. 262, no. 1, pp. 12-18, 1999.

[69] E. P. Sablin, R. B. Case, S. C. Dai et al., "Direction determination in the minus-end-directed kinesin motor ncd," Nature, vol. 395, no. 6704, pp. 813-816, 1998.

[70] R. Mallik and S. P. Gross, "Molecular motors: strategies to get along," Current Biology, vol. 14, no. 22, pp. R971-R982, 2004.

[71] M. Schliwa and G. Woehlke, "Molecular motors," Nature, vol. 422, no. 6933, pp. 759-765, 2003.

[72] S. L. Anderson, R. Coli, I. W. Daly et al., "Familial dysautonomia is caused by mutations of the IKAP gene," American Journal of Human Genetics, vol. 68, no. 3, pp. 753-758, 2001.

[73] C. L. Simpson, R. Lemmens, K. Miskiewicz et al., "Variants of the elongator protein 3 (ELP3) gene are associated with motor neuron degeneration," Human Molecular Genetics, vol. 18, no. 3, pp. 472-481, 2009.

[74] F. B. Axelrod, "Familial dysautonomia," Muscle and Nerve, vol. 29, no. 3, pp. 352-363, 2004.

[75] M. P. Cuajungco, M. Leyne, J. Mull et al., "Tissue-specific reduction in splicing efficiency of IKBKAP due to the major mutation associated with familial dysautonomia," American Journal of Human Genetics, vol. 72, no. 3, pp. 749-758, 2003.

[76] K. J. De vos, A. L. Chapman, M. E. Tennant et al., "Familial amyotrophic lateral sclerosis-linked SOD1 mutants perturb fast axonal transport to reduce axonal mitochondria content," Human Molecular Genetics, vol. 16, no. 22, pp. 2720-2728, 2007.

[77] A. R. Saha, J. Hill, M. A. Utton et al., "Parkinson's disease $\alpha$-synuclein mutations exhibit defective axonal transport in cultured neurons," Journal of Cell Science, vol. 117, no. 7, pp. 1017-1024, 2004.

[78] G. B. Stokin, C. Lillo, T. L. Falzone et al., "Axonopathy and transport deficits early in the pathogenesis of Alzheimer's diseases," Science, vol. 307, no. 5713, pp. 1282-1288, 2005.

[79] J. W. Hammond, D. Cai, and K. J. Verhey, "Tubulin modifications and their cellular functions," Current Opinion in Cell Biology, vol. 20, no. 1, pp. 71-76, 2008.

[80] B. D. Strahl and C. D. Allis, "The language of covalent histone modifications," Nature, vol. 403, no. 6765, pp. 41-45, 2000. 

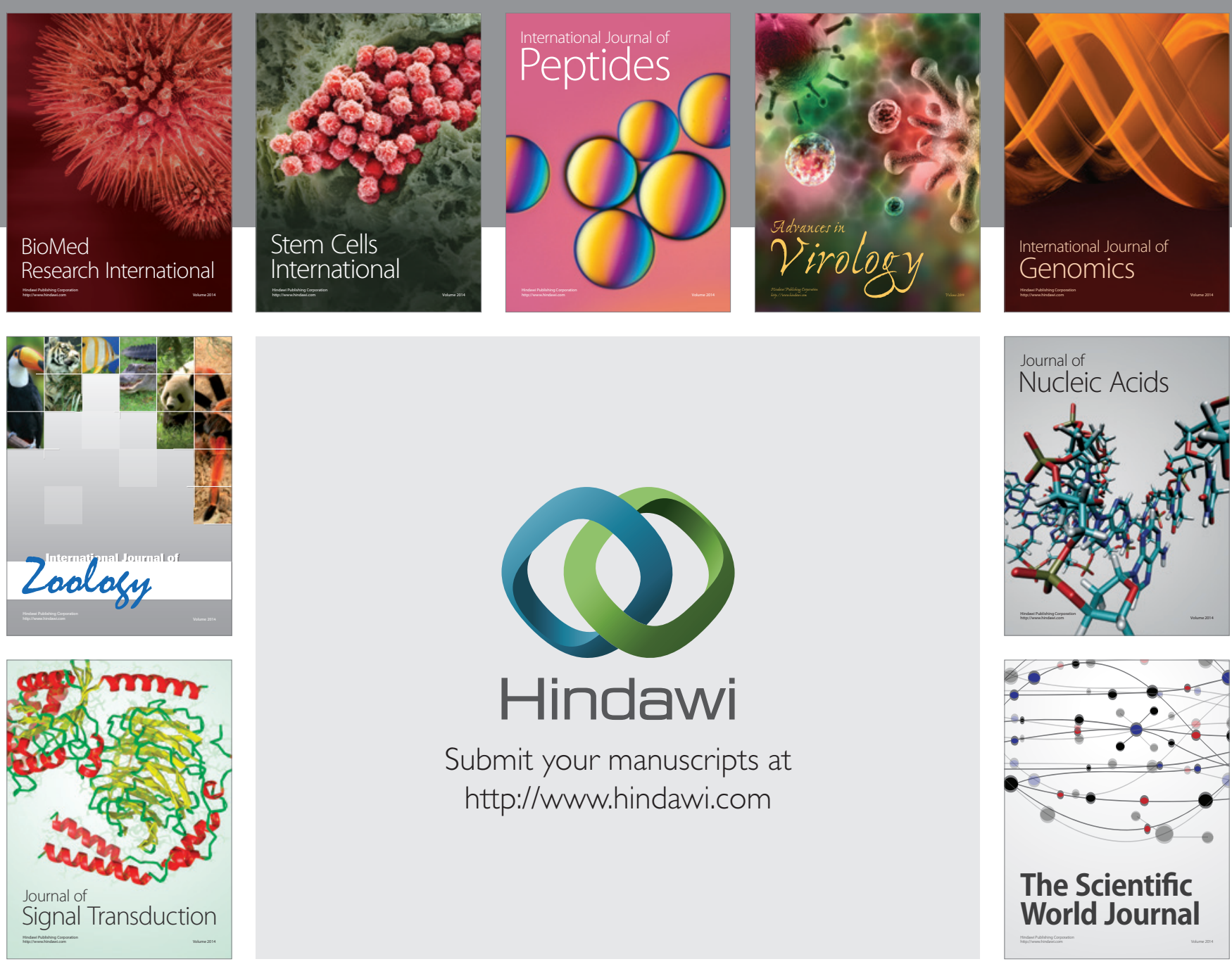

Submit your manuscripts at

http://www.hindawi.com
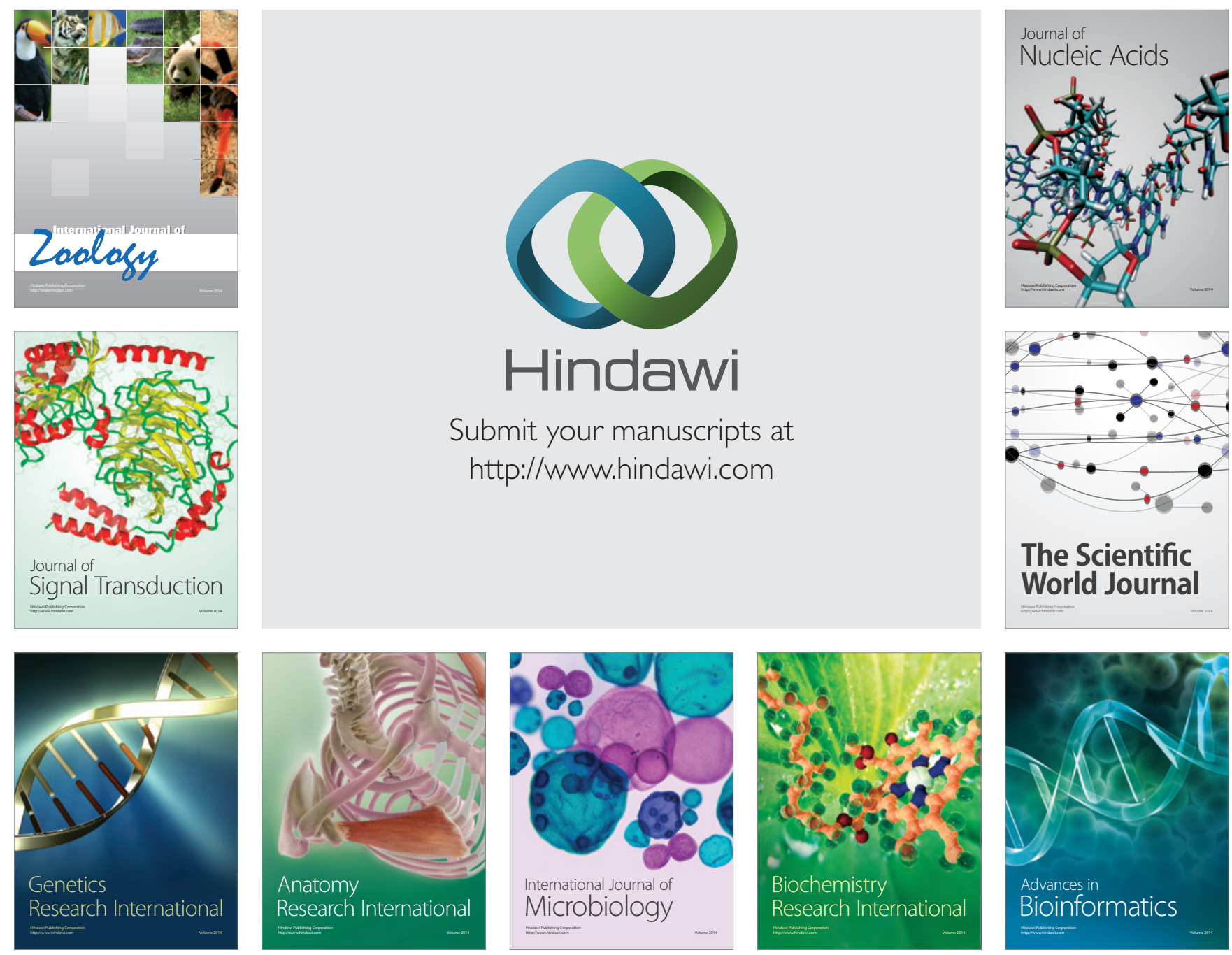

The Scientific World Journal
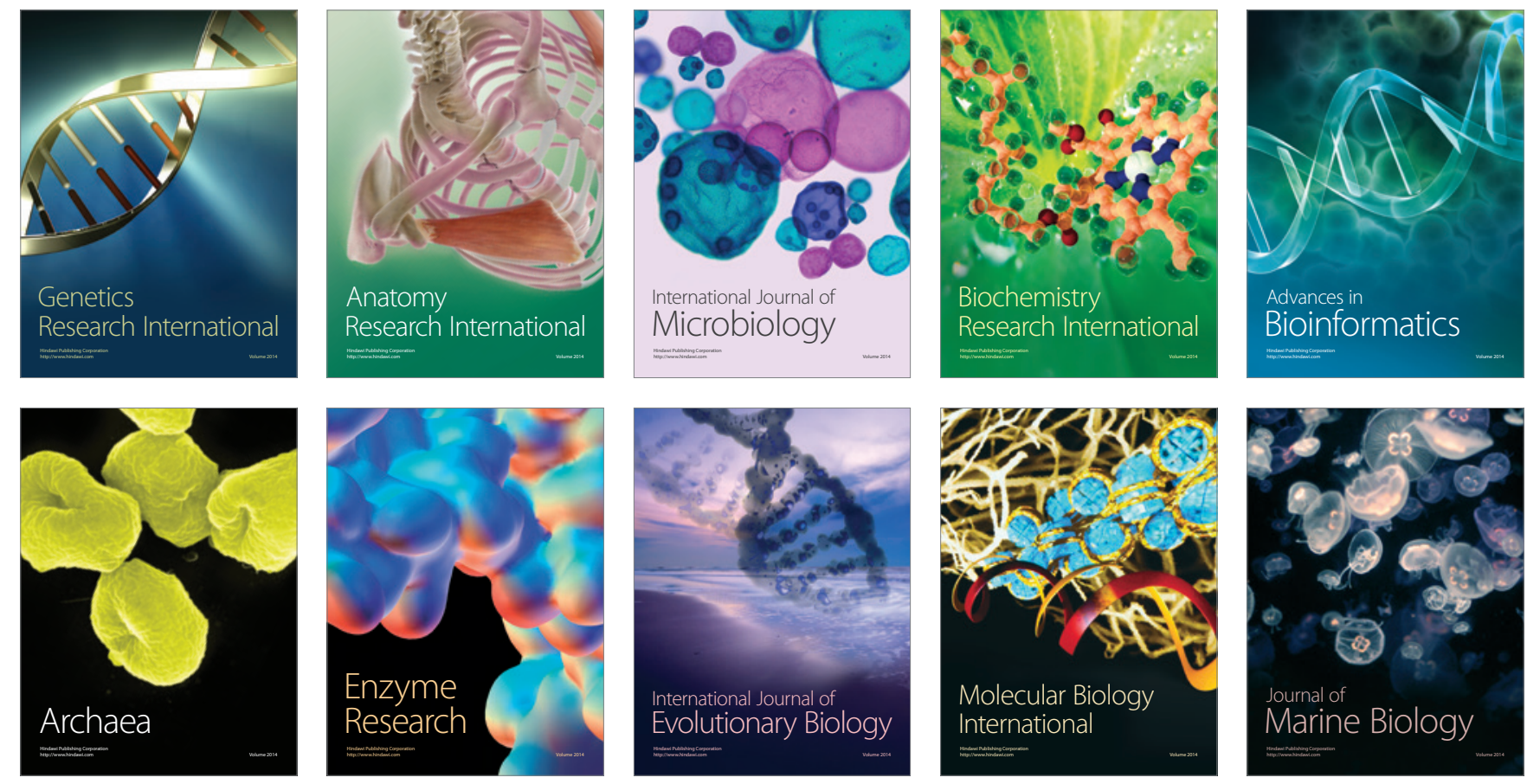